\title{
High cumulative doxorubicin dose for advanced soft tissue sarcoma
}

\author{
Zhichao Tian ${ }^{1 *} \mathbb{D}$, Yang Yang ${ }^{2}$, Yonghao Yang ${ }^{3}$, Fan Zhang ${ }^{1}$, Po Li $i^{1}$, Jiaqiang Wang ${ }^{1}$, Jinpo Yang ${ }^{4}$, Peng Zhang ${ }^{1}$, \\ Weitao Yao ${ }^{1}$ and Xin Wang ${ }^{1}$
}

\begin{abstract}
Background: The recommended cumulative doxorubicin dose in soft tissue sarcoma (STS) treatment was based on cardiotoxicity data from retrospective studies of breast cancer patients. However, the treatment and prognosis of STS and breast cancer are quite different, and reference to breast cancer data alone may not reflect the efficacy of doxorubicin treatment in STS. This study, thus, aimed to review and analyze clinical data of STS patients treated with a high cumulative doxorubicin dose, to provide a reference for treatment selection and clinical trial design.

Methods: We retrospectively collected and analyzed clinical data of patients with advanced STS who received doxorubicin-based chemotherapy from January 2016 to January 2020. The patients were divided into a standard-dose group (who received $\leq 6$ cycles of doxorubicin after the initial diagnosis) and an over-dose group (who were re-administered doxorubicin [doxorubicin-rechallenge] after receiving 6 cycles of doxorubicin therapy discontinuously). Patient characteristics, cumulative doxorubicin dose, objective response rate (ORR), disease control rate (DCR), progression-free survival (PFS), cardiotoxicity incidence, and treatment effectiveness were evaluated in both groups.
\end{abstract}

Results: A total of 170 patients with advanced STS were recruited (146 in the standard-dose group and 24 in the over-dose group). The average cumulative doxorubicin dose was $364.04 \pm 63.81 \mathrm{mg} / \mathrm{m} 2$ in the standarddose group and $714.38 \pm 210.09 \mathrm{mg} / \mathrm{m} 2$ in the over-dose group. The ORR, DCR, and median PFS were 15.07, 58.9\%, and 6 (95\% confidence interval [Cl]: 5.8-6.5) months in the standard-dose group and 16.67, 66.67\%, and 4 (95\%Cl: 2.0-5.8) months in the over-dose group, respectively. Symptomatic heart failure occurred in five patients (3.42\%) of the standard-dose group and in one patient $(4.17 \%)$ of the over-dose group. In these patients with cardiotoxicity, doxorubicin was discontinued, and all of them died of uncontrolled tumor growth. No drug-related deaths occurred.

Conclusions: The continuation of or rechallenge with doxorubicin beyond the recommended cumulative dose could be a promising therapeutic option in the treatment of chemotherapy-sensitive advanced sarcomas. Further evaluation is necessary in prospective trials.

Keywords: Soft-tissue sarcoma, Doxorubicin, Dexrazoxane, Cardiotoxicity, Cumulative dose

\footnotetext{
* Correspondence: tianzhichaoyy@163.com

'Department of Bone and Soft Tissue, the Affiliated Cancer Hospital of

Zhengzhou University and Henan Cancer Hospital, Dongming road,

Zhengzhou 450008, Henan Province, China

Full list of author information is available at the end of the article
}

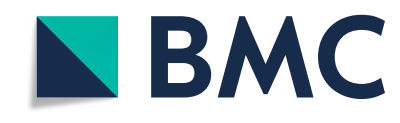

(c) The Author(s). 2020 Open Access This article is licensed under a Creative Commons Attribution 4.0 International License, which permits use, sharing, adaptation, distribution and reproduction in any medium or format, as long as you give appropriate credit to the origin author(s) and the source, provide a link to the Creative Commons licence, and indicate if changes were made. The images or other third party material in this article are included in the article's Creative Commons licence, unless indicated otherwise in a credit line to the material. If material is not included in the article's Creative Commons licence and your intended use is not permitted by statutory regulation or exceeds the permitted use, you will need to obtain permission directly from the copyright holder. To view a copy of this licence, visit http://creativecommons.org/licenses/by/4.0/. The Creative Commons Public Domain Dedication waiver (http://creativecommons.org/publicdomain/zero/1.0/) applies to the data made available in this article, unless otherwise stated in a credit line to the data. 


\section{Background}

As a type of anthracycline, doxorubicin has long been used in chemotherapy for malignancies. At present, doxorubicin is used as a standard chemotherapeutic drug for breast cancer, ovarian cancer, sarcoma, lymphoma, small-cell lung cancer, and many other malignant tumors [1]. Its efficacy in these malignancies has been repeatedly confirmed. However, continuous use of doxorubicin in treatable patients is associated with dosedependent cardiotoxicity [2]. The initial study of the drug identified a $3 \%$ incidence of cardiotoxicity (mainly heart failure) at a cumulative dose of $400 \mathrm{mg} / \mathrm{m}^{2}, 7 \%$ at $550 \mathrm{mg} / \mathrm{m}^{2}$, and $18 \%$ at $700 \mathrm{mg} / \mathrm{m}^{2}$ [3]. Another study showed that the incidence of cardiotoxicity in 630 patients treated with doxorubicin was $5.1 \%$ at a mean cumulative doxorubicin dose of $283 \mathrm{mg} / \mathrm{m}^{2}$ [4]. In other studies, the incidence of doxorubicin-induced cardiotoxicity varied greatly due to the different definitions of cardiotoxicity used $[5,6]$. Despite these differences in findings, it is generally accepted that the cardiotoxicity of doxorubicin increases with cumulative dose. Thus, the optimal cumulative dose of doxorubicin remains a key concern in the treatment of many malignancies. The currently accepted safe cumulative dose of doxorubicin is $<450 \mathrm{mg} / \mathrm{m}^{2}$ [7-9].

Soft-tissue sarcoma (STS) is a rare malignancy. However, there are still about 40,000 new cases of STS in China every year [10]. About half of these are at the advanced-disease stage or will eventually progress to advanced disease. The first-line treatment for advanced STS is doxorubicin-based chemotherapy [11-13]. However, due to the low incidence of sarcomas, the study of doxorubicin-induced cardiotoxicity is far less common in sarcoma patients than in breast cancer patients. Although the cumulative doxorubicin dose was reported to be significantly higher for sarcoma than for breast cancer, there was no corresponding increase in the incidence of cardiotoxicity in sarcoma [14-16]. In the treatment of STS, the recommended cumulative doxorubicin dose is also $450 \mathrm{mg} / \mathrm{m}^{2}$ [13], which is clearly based on data for other malignancies, particularly breast cancer [17]. However, the treatment and prognosis of STS and breast cancer are quite different, and reference to breast cancer data alone may not reflect or improve the efficacy of doxorubicin treatment in STS.

As a major sarcoma treatment center in central China, our institution has used high cumulative doses of doxorubicin in our clinical work to treat a number of patients with advanced STS, because of the lack of other effective treatment modalities [18]. In this study, we aimed to review and analyze the clinical data of these patients to provide a reference for treatment selection and clinical trial design for treating advanced STS.

\section{Methods}

\section{Patients and eligibility criteria}

To provide reference criteria for the selection of a treatment dose and to aid in identifying factors for future clinical trials studying doxorubicin dose in STS, we retrospectively collected and analyzed the clinical data of patients with advanced STS who received doxorubicinbased chemotherapy at the Affiliated Cancer Hospital of Zhengzhou University from January 2016 to January 2020. The inclusion criteria were as follows: (1) patients who were pathologically diagnosed with advanced-stage STS, (2) patients who received at least 2 cycles of doxorubicin-based chemotherapy, and [19] patients with complete clinical and follow-up data.

\section{Statistical indicators and analysis}

According to the cumulative dose and number of cycles of doxorubicin, the patients were divided into two groups: the standard cumulative dose group (standarddose group) and the over-cumulative dose group (overdose group). The standard-dose group comprised patients who received $\leq 6$ cycles of doxorubicin after the initial diagnosis, while the over-dose group consisted of patients who were re-administered doxorubicin (doxorubicin rechallenge) after the discontinuation of doxorubicin therapy (6 cycles) as other treatment methods (other chemotherapeutic agents, tyrosine kinase inhibitors or immune checkpoint inhibitors) were ineffective. The effectiveness and cardiotoxicity of doxorubicin therapy in the first treatment phase with 6 cycles (the pre-over-dose group) were also assessed.

The general characteristics, cumulative doxorubicin dose, objective response rate (ORR), disease control rate (DCR), progression-free survival (PFS), and incidence of cardiotoxicity in the two groups were assessed. Treatment effectiveness was evaluated according to the Response Evaluation Criteria in Solid Tumors version 1.1. The baseline therapeutic effectiveness in the overdose group was based on the target lesion diameter at the beginning of the doxorubicin rechallenge. PFS in the standard-dose group and the pre-over-dose group was defined as the time from the start of doxorubicin treatment to the occurrence of progressive disease or to the switch to another treatment regimen. PFS in the overdose group was defined as the time from the start of the doxorubicin rechallenge to the occurrence of progressive disease or death. Cardiotoxicity was defined as heart failure with the associated symptoms and clinical signs.

All continuous variables are presented as means and standard deviations, and all categorical variables are expressed as numbers and percentages of patients. Survival analysis was performed using the Kaplan-Meier method with a 95\% confidence interval (CI). Figures were drawn using GraphPad Prism 5.0 (GraphPad 
Software Inc., San Diego, CA, USA). All statistical analyses were performed using SPSS software version 24.0 for Windows (IBM Corp., Armonk, NY, USA).

\section{Results}

\section{Patient characteristics}

A total of 170 patients with advanced STS were recruited. There were 146 patients in the standard-dose group and 24 in the over-dose group. The general characteristics of these patients are shown in Table 1.

Table 1 Patient characteristics of standard-dose group and over-dose group

\begin{tabular}{|c|c|c|}
\hline Characteristics & $\begin{array}{l}\text { Standard-dose } \\
\text { group }(n=146)\end{array}$ & $\begin{array}{l}\text { Over-dose } \\
\text { group }(n=24)\end{array}$ \\
\hline \multicolumn{3}{|l|}{ Gender } \\
\hline Male & $70(47.95 \%)$ & $14(58.33 \%)$ \\
\hline Female & $76(52.05 \%)$ & $10(41.67 \%)$ \\
\hline Age & $43.30 \pm 12.10$ & $38.58 \pm 14.01$ \\
\hline \multicolumn{3}{|l|}{ ECOG PS } \\
\hline 0 & $71(48.63 \%)$ & $7(29.17 \%)$ \\
\hline 1 & $75(51.37 \%)$ & $17(70.83 \%)$ \\
\hline \multicolumn{3}{|l|}{ Histological types } \\
\hline Undifferentiated sarcoma & $36(24.66 \%)$ & $6(25.00 \%)$ \\
\hline Synovial sarcoma & $23(15.75 \%)$ & $4(16.67 \%)$ \\
\hline Leiomyosarcoma & $20(13.70 \%)$ & $3(12.50 \%)$ \\
\hline Fibrosarcoma & $16(10.96 \%)$ & $3(10.34 \%)$ \\
\hline Liposarcoma & $15(10.27 \%)$ & $2(8.33 \%)$ \\
\hline Angiosarcoma & $14(9.59 \%)$ & $6(25.00 \%)$ \\
\hline Epithelioid sarcoma & $7(4.79 \%)$ & \\
\hline MPNST & $7(4.79 \%)$ & \\
\hline Clear cell sarcoma & $3(2.05 \%)$ & \\
\hline Others & $5(3.42 \%)$ & \\
\hline \multicolumn{3}{|c|}{ Metastatic or locally unresectable } \\
\hline Locally unresectable & $20(13.70 \%)$ & $7(29.17 \%)$ \\
\hline Metastatic & $120(82.20 \%)$ & $17(70.83 \%)$ \\
\hline Both & $6(4.11 \%)$ & 0 \\
\hline \multicolumn{3}{|l|}{ Primary site } \\
\hline Extremities & $101(69.18 \%)$ & $14(58.33 \%)$ \\
\hline Trunk & $45(30.82 \%)$ & $10(41.67 \%)$ \\
\hline \multicolumn{3}{|l|}{ Metastatic site } \\
\hline Lung & $124(84.93 \%)$ & $16(66.67 \%)$ \\
\hline Other & $22(15.07 \%)$ & $8(33.33 \%)$ \\
\hline $\begin{array}{l}\text { Mean cycles of doxorubicin } \\
\text { chemotherapy }\end{array}$ & 5 & 11.5 \\
\hline
\end{tabular}

Notes: Data are presented as numbers (percentages) or means \pm standard deviations

Abbreviations: ECOG PS Eastern Cooperative Oncology Group performance status, MPNST malignant peripheral nerve sheath tumor
The youngest patient was aged 17 years, and the oldest was 65 years. The mean age of the standard-dose group was $43.30 \pm 12.10$ years, while that of the over-dose group was $38.58 \pm 14.01$ years. Physical fitness was good in both groups, and no patients had an Eastern Cooperative Oncology Group performance status score of $\geq 2$. The most common histopathological subtypes were undifferentiated sarcoma, synovial sarcoma, and leiomyosarcoma in the standard-dose group, and undifferentiated sarcoma, synovial sarcoma, and angiosarcoma in the over-dose group. Most of the patients in both groups had primary lesions in their extremities and lung metastases. Patients in the standarddose group received an average of 5 cycles of doxorubicin, while those in the over-dose group received an average of 11.5 cycles.

\section{Treatment and dose modification}

The treatment of patients and the study flowchart used in this study are shown in Fig. 1. All patients received doxorubicin at an initial dose of $37.5 \mathrm{mg} / \mathrm{m}^{2}$ via intravenous bolus injection on days 1 and 2 of a 21-day cycle. All patients were also treated with dexrazoxane at the beginning of the doxorubicin treatment. In the standard-dose group, the dose of doxorubicin was modestly reduced in patients with severe adverse events (all of which were hemotoxic), and doxorubicin was administered until disease progression or until 6 cycles were completed. Doxorubicin rechallenge was undertaken in the patients in the over-dose group. In other words, doxorubicin was discontinued in these patients after they received 6 cycles of doxorubicin therapy and then restarted after other treatment methods proved ineffective. The average cumulative doxorubicin dose was $364.04 \pm 63.81 \mathrm{mg} / \mathrm{m}^{2}$ in the standard-dose group and $714.38 \pm 210.09 \mathrm{mg} / \mathrm{m}^{2}$ in the over-dose group (Table 2, Fig. 2). The average cumulative doxorubicin dose in the pre-over-dose group was $421.15 \pm$ $47.39 \mathrm{mg} / \mathrm{m}^{2}$ (Table 2 ).

\section{Effectiveness}

One patient with angiosarcoma in the over-dose group achieved a complete response. In the standard-dose group, the ORR, DCR, and median PFS were 15.07, $58.9 \%$, and 6 (95\% CI: 5.8-6.5) months, respectively (Table 2, Fig. 3). In the pre-overdose group, the ORR, DCR, and median PFS were 100, 100\%, and 8.15 (95\% CI: $5.8-9.5$ ) months, respectively (Table 2, Fig. 3). In the over-dose group, the ORR, DCR, and median PFS were 16.67, 66.67\%, and 4 (95\% CI: 2.0-5.8) months, respectively (Table 2, Fig. 3). 


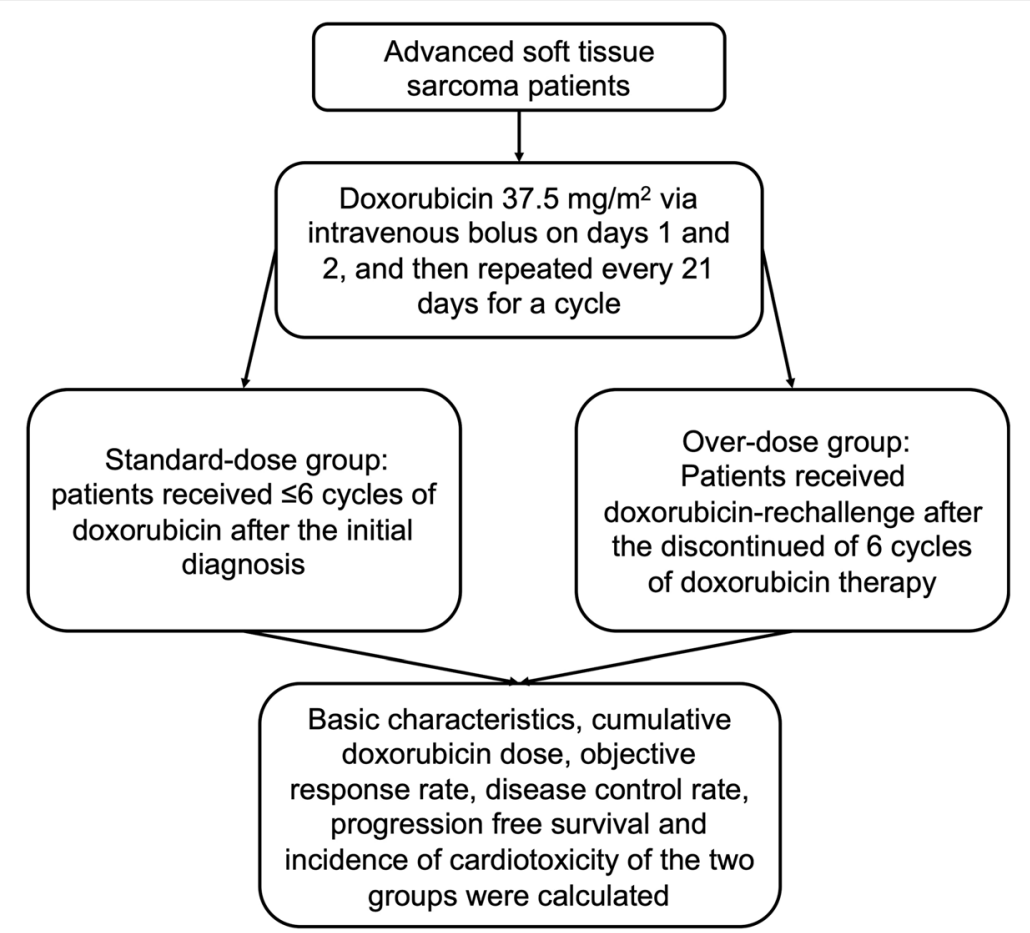

Fig. 1 The treatment of patients and the study flowchart used in this study

\section{Cardiotoxicity}

The incidence of cardiotoxicity in both groups was low (Table 2). Five patients (3.42\%) developed symptomatic heart failure in the standard-dose group (Table 2, Fig. 2), whereas only one patient $(4.17 \%)$ had symptomatic heart failure in the over-dose group (Table 2, Fig. 2). Owing to cardiotoxicity, these patients stopped doxorubicin, and all died of uncontrolled tumor growth. No drug-related deaths were noted in this study.

\section{Discussion}

In this study, we evaluated the ORR, DCR, PFS, and cardiotoxicity of doxorubicin in patients with advanced STS. Patients in the standard-dose group, who received $\leq 6$ cycles of doxorubicin after the initial diagnosis, had an average cumulative doxorubicin dose of 364.04 \pm $63.81 \mathrm{mg} / \mathrm{m}^{2}$. Patients in the over-dose group had been administered 6 cycles of doxorubicin, and were administered doxorubicin again for various reasons; the average cumulative doxorubicin dose in this group was $714.38 \pm 210.09 \mathrm{mg} / \mathrm{m}^{2}$. The standard-dose group was assessed in first line, whereas the over-dose group was assessed in further-line ( $>1$ treatment line). Because of the significant differences in the cumulative dose and timing of doxorubicin and other medication conditions between the groups, as well as the non-randomness of the enrolled cases and the retrospective nature of this study, we did not conduct a statistical comparative test on the two groups of data. Nevertheless, according to the results, in some patients with advanced STS, treatment with an over-cumulative dose of doxorubicin (or rechallenge with doxorubicin beyond the recommended cumulative dose) led to remarkable efficacy without an increase in cardiotoxicity.

Cardiotoxicity in this study was simply defined as the presence of heart failure, with its associated symptoms

Table 2 Clinical characteristics, effectiveness and safety of the two groups

\begin{tabular}{llll}
\hline Characteristics & Standard-dose group $(\boldsymbol{n}=\mathbf{1 4 6})$ & Pre-over-dose group $(\boldsymbol{n}=\mathbf{2 4})$ & Over-dose group $(\boldsymbol{n}=\mathbf{2 4})$ \\
\hline Accumulative Doxorubicin dose $\left(\mathrm{mg} / \mathrm{m}^{2}\right)$ & $364.04 \pm 63.81$ & $421.15 \pm 47.39$ & $714.38 \pm 210.09$ \\
ORR (\%) & $15.07 \%$ & $100 \%$ & $16.67 \%$ \\
DCR (\%) & $58.9 \%$ & $100 \%$ & $66.67 \%$ \\
Median-PFS (months) & $6(95 \% \mathrm{Cl}: 5.8-6.5)$ & $8.15(95 \% \mathrm{Cl}: 5.8-9.5)$ & $4(95 \% \mathrm{Cl}: 2.0-5.8)$ \\
Incidence of cardiotoxicity (\%) & $3.42 \%$ & $0 \%$ & $4.17 \%$ \\
\hline
\end{tabular}

Notes: Data are presented as numbers (percentages) or means \pm standard deviations

Abbreviations: ORR objective response rate, DCR disease control rate, Median-PFS median progression-free survival 


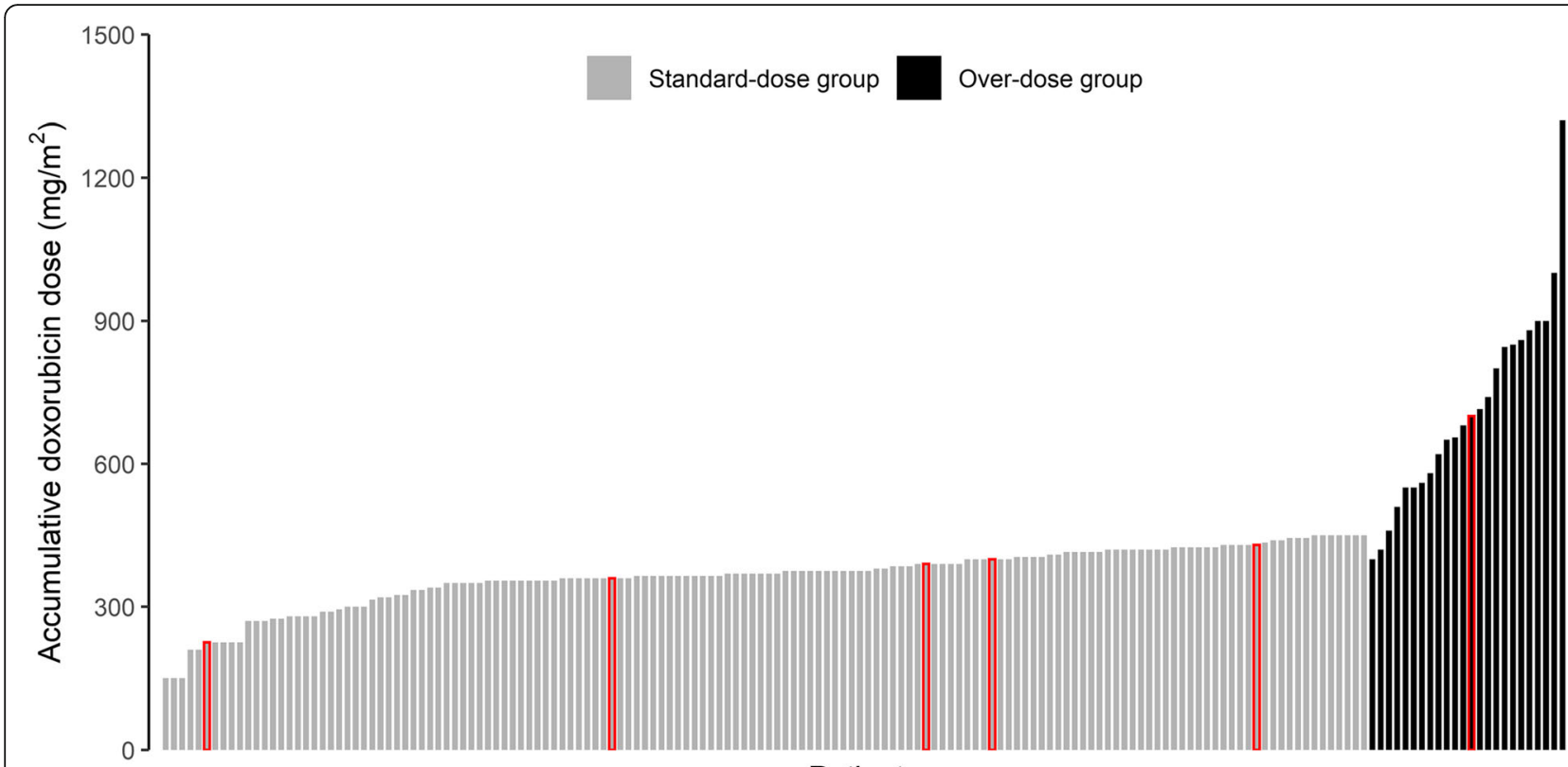

Patients

Fig. 2 cumulative doxorubicin dose for patients in this study. In red circles are patients who have developed cardiotoxicity

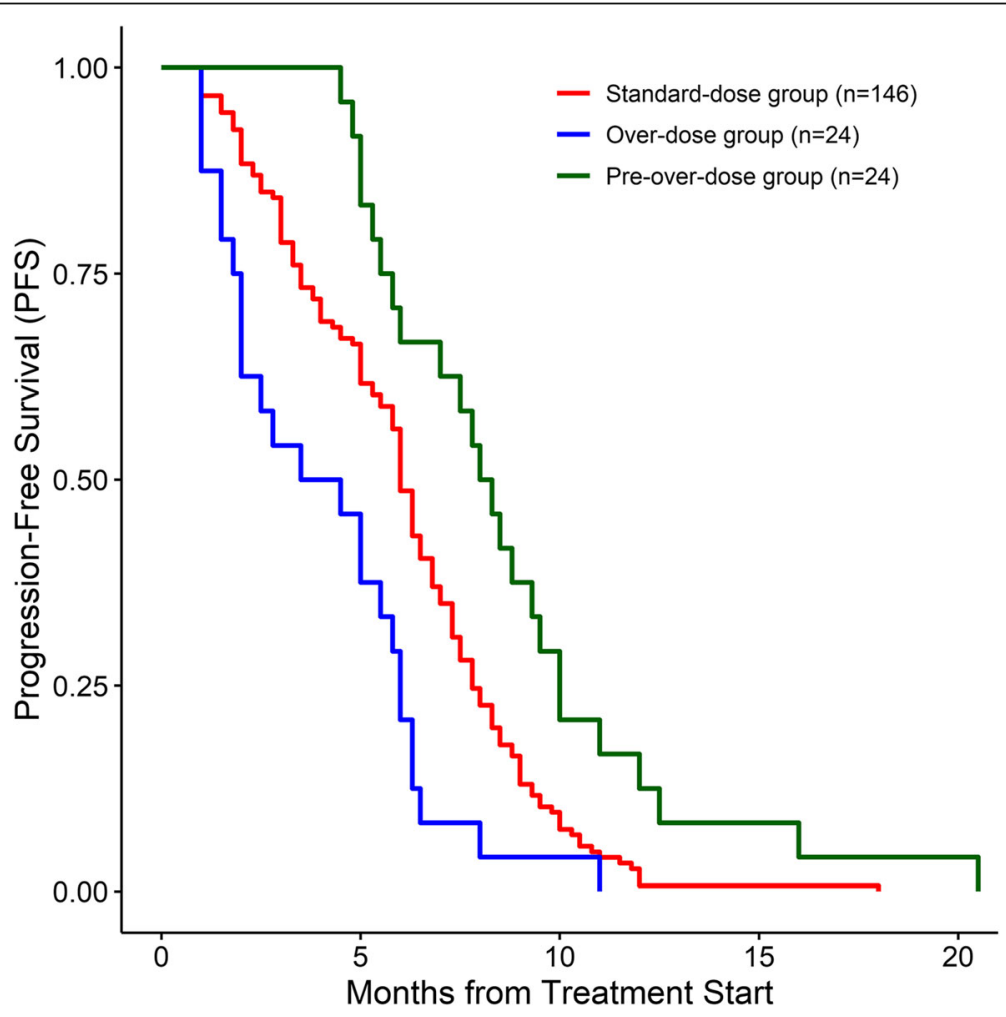

Fig. 3 progression-free survival among patients with advanced STS in this study 
and clinical signs. There are several definitions of doxorubicin-associated cardiotoxicity, and these definitions are significantly different from each other and based on different incidence rates $[4,20]$. With the invention of new detection methods and the improvement of detection sensitivity, researchers have observed cardiotoxicity in low cumulative doses of doxorubicin [21]. A broad definition of cardiotoxicity may make sense for patients with long-term survival, such as breast cancer patients, because of the risk of long-term cardiac disease in these patients [1]. It is clear, however, that for patients with advanced STS with limited survival, the most significant definition of cardiotoxicity would be symptomatic heart failure, as asymptomatic heart damage is of little significance for these patients.

Several studies have proven that a high cumulative dose of doxorubicin is only one of the factors leading to cardiotoxicity. Other factors that can significantly increase the risk of cardiotoxicity include older age ( $>65$ years), metastasis, infusion regimen, hypertension, overweight, preexisting cardiac disease, concomitant use of other drugs, and pericardial radiotherapy $[8,20,22,23]$. In this study, there were fewer risk factors for cardiotoxicity in both groups of patients, especially in the over-dose group. Some high-risk patients might have already developed cardiotoxicity during screening, so they were excluded from the over-dose group. Furthermore, we might have unintentionally excluded high-risk patients during patient selection. These could have contributed to the lack of significant increase in the incidence of cardiotoxicity in the over-dose group in this study. Similar to the results of several other studies $[8,24]$, the cumulative doxorubicin dose in patients with cardiotoxicity in this study was not the highest among the patients, which indicates that the cumulative doxorubicin dose was not the only factor leading to cardiotoxicity.

In addition, dexrazoxane could also be a reason why there was no significant increase in the incidence of cardiotoxicity in the over-dose group in this study. Although dexrazoxane does not completely eliminate doxorubicin cardiotoxicity $[21,25]$, it is still an option for primary prevention to reduce the risk of doxorubicin cardiotoxicity [7, 25]. Published data have shown that patients with sarcoma can receive a mean cumulative doxorubicin dose of $600-750 \mathrm{mg} / \mathrm{m}^{2}$ without a significant increase in cardiotoxicity when they are administered dexrazoxane $[15,16]$. Similar to the above studies, this study is also confirmed that dexrazoxane can reduce cardiotoxicity in doxorubicin-treated sarcoma patients.

There are several guidelines regarding the management of doxorubicin-related cardiotoxicity [9, 26]. These guidelines generally provide an upper limit for the cumulative doxorubicin dose based on cardiotoxicity data from retrospective studies of breast cancer patients, but are limited in that they do not consider some specific patients. In these specific patients, the benefits of a high cumulative doxorubicin dose may outweigh its costs. The results of this study suggest that such ideas and guidelines should be reviewed and revised. The cumulative dose of doxorubicin is a factor that needs to be considered in individualized treatment, but it should not be a limiting factor. Patients with advanced STS have fewer effective treatments and a shorter survival than patients with breast cancer. Doses above the standard upper limit of the cumulative dose of doxorubicin at a certain stage (when no other effective treatment is available) should be administered in patients with advanced STS who are in a good physical condition or otherwise, if doxorubicin remains effective.

Although the small sample size and the retrospective design of this study affect the credibility of the results, the results obtained suggest that the continuation or rechallenge with doxorubicin beyond the recommended cumulative dose is a promising option to improve survival in patients with advanced STS. Nevertheless, further evaluation through prospective trials is necessary to validate these findings. Early monitoring of doxorubicinrelated cardiotoxicity is also worthy of further study.

\section{Conclusions}

A high cumulative dose of doxorubicin for advanced STS was associated with a better efficacy. Moreover, there was no significant increase in cardiotoxicity. The continuation or rechallenge with doxorubicin beyond the recommended cumulative dose could be a promising therapeutic option in the treatment of chemotherapysensitive advanced STS. Further evaluation in prospective trials is therefore warranted.

\section{Abbreviations \\ Cl: Confidence interval; DCR: Disease control rate; ECOG PS: Eastern Cooperative Oncology Group performance status; MPNST: Malignant peripheral nerve sheath tumor; ORR: Objective response rate; \\ PFS: Progression-free survival; STS: Soft tissue sarcoma}

\section{Acknowledgements}

We thank all Chinese patients and investigators who participated in this study.

\section{Authors' contributions}

All of the authors have made substantial contributions to the conception. Conception and design: ZT and WY. Patient recruitment and acquisition of data: $Z T, Y Y 1, Y Y 2, F Z, P L, J W, J Y, P Z$ and $X W$. Analysis and interpretation of data: $Y Y 1$ and FZ. Writing of the manuscript: ZT. Review and revision of the manuscript: $Y Y 1, Y Y 2, F Z, P L, J W, J Y, P Z, W Y$ and $X W$. Study supervision: JW, $P Z, W Y$ and $X W$. All authors have read and approved the manuscript.

\section{Funding}

Not applicable.

\section{Availability of data and materials}

The datasets used and/or analysed during the current study are available from the corresponding author on reasonable request. 


\section{Ethics approval and consent to participate}

The clinical data was acquired with the approval and permission of the Ethics Committee of The Affiliated Cancer Hospital of Zhengzhou University. The study protocol was approved by the Ethics Committee of The Affiliated Cancer Hospital of Zhengzhou University. The study was conducted in accordance with the Declaration of Helsinki. All patients provided written informed consent

\section{Consent for publication}

Not applicable.

\section{Competing interests}

The authors declare they have no competing interests.

\section{Author details}

${ }^{1}$ Department of Bone and Soft Tissue, the Affiliated Cancer Hospital of Zhengzhou University and Henan Cancer Hospital, Dongming road, Zhengzhou 450008, Henan Province, China. ${ }^{2}$ Huanghe Science and Technology College, Zhengzhou 450063, Henan Province, China. ${ }^{3}$ Department of Immunotherapy, The Affiliated Cancer Hospital of Zhengzhou University and Henan Cancer Hospital, Zhengzhou 450008, Henan Province, China. ${ }^{4}$ Department of Medical Oncology, the Affiliated Cancer Hospital of Zhengzhou University and Henan Cancer Hospital, Zhengzhou 450008, Henan Province, China.

\section{Received: 26 August 2020 Accepted: 18 November 2020}

Published online: 23 November 2020

\section{References}

1. McGowan JV, Chung R, Maulik A, Piotrowska I, Walker JM, Yellon DM. Anthracycline chemotherapy and Cardiotoxicity. Cardiovasc Drugs Ther. 2017;31(1):63-75.

2. Henriksen PA. Anthracycline cardiotoxicity: an update on mechanisms, monitoring and prevention. Heart. 2018;104(12):971-7.

3. Von Hoff DD, Layard MW, Basa P, Davis HL, Von Hoff AL, Rozencweig M, Muggia FM. Risk factors for doxorubicin-induced congestive heart failure. Ann Intern Med. 1979;91(5):710-7.

4. Swain SM, Whaley FS, Ewer MS. Congestive heart failure in patients treated with doxorubicin: a retrospective analysis of three trials. Cancer. 2003;97(11):2869-79.

5. Swain SM, Whaley FS, Gerber MC, Weisberg S, York M, Spicer D, Jones SE, Wadler S, Desai A, Vogel C, et al. Cardioprotection with dexrazoxane for doxorubicin-containing therapy in advanced breast cancer. J Clin Oncol. 1997;15(4):1318-32.

6. Singal PK, lliskovic N. Doxorubicin-induced cardiomyopathy. N Engl J Med. 1998;339(13):900-5.

7. Upshaw JN. Cardioprotective strategies to prevent Cancer treatment-related cardiovascular toxicity: a review. Curr Oncol Rep. 2020;22(7):72.

8. Cho H, Lee S, Sim SH, Park IH, Lee KS, Kwak MH, Kim HJ. Cumulative incidence of chemotherapy-induced cardiotoxicity during a 2-year follow-up period in breast cancer patients. Breast Cancer Res Treat. 2020;182(2):333-43

9. Zamorano JL, Lancellotti P, Rodriguez Munoz D, Aboyans V, Asteggiano R, Galderisi M, Habib G, Lenihan DJ, Lip GY, Lyon AR, et al. 2016 ESC position paper on cancer treatments and cardiovascular toxicity developed under the auspices of the ESC Committee for practice guidelines: the task force for cancer treatments and cardiovascular toxicity of the European Society of Cardiology (ESC). Eur J Heart Fail. 2017;19(1):9-42.

10. Yang Z, Zheng R, Zhang S, Zeng H, Li H, Chen W. Incidence, distribution of histological subtypes and primary sites of soft tissue sarcoma in China. Cancer Biol Med. 2019;16(3):565-74

11. Gronchi A, Palmerini E, Quagliuolo V, et al. Neoadjuvant chemotherapy in high-risk soft tissue sarcomas: final results of a randomized trial from Italian (ISG), Spanish (GEIS), French (FSG), and polish (PSG) sarcoma groups. J Clin Oncol. 2020;38(19):2178-86.

12. Judson I, Verweij J, Gelderblom H, Hartmann JT, Schöffski P, Blay J-Y, Kerst JM, Sufliarsky J, Whelan J, Hohenberger P, et al. Doxorubicin alone versus intensified doxorubicin plus ifosfamide for first-line treatment of advanced or metastatic soft-tissue sarcoma: a randomised controlled phase 3 trial. Lancet Oncol. 2014;15(4):415-23.
13. Seddon B, Strauss SJ, Whelan J, Leahy M, Woll PJ, Cowie F, Rothermundt C, Wood Z, Benson C, Ali N, et al. Gemcitabine and docetaxel versus doxorubicin as first-line treatment in previously untreated advanced unresectable or metastatic soft-tissue sarcomas (GeDDiS): a randomised controlled phase 3 trial. Lancet Oncol. 2017;18(10):1397-410.

14. Shamai S, Rozenbaum Z, Merimsky O, Derakhshesh M, Moshkovits Y, Arnold J, Topilsky Y, Arbel Y, Laufer-Perl M. Cardio-toxicity among patients with sarcoma: a cardio-oncology registry. BMC Cancer. 2020;20(1):609.

15. Schwartz CL, Wexler LH, Krailo MD, Teot LA, Devidas M, Steinherz LJ, Goorin AM, Gebhardt MC, Healey JH, Sato JK, et al. Intensified chemotherapy with Dexrazoxane Cardioprotection in newly diagnosed nonmetastatic osteosarcoma: a report from the Children's oncology group. Pediatr Blood Cancer. 2016;63(1):54-61.

16. Schuler MK, Gerdes S, West A, Richter S, Busemann C, Hentschel L, Lenz $F$, Kopp HG, Ehninger $G$, Reichardt $P$, et al. Efficacy and safety of Dexrazoxane (DRZ) in sarcoma patients receiving high cumulative doses of anthracycline therapy - a retrospective study including 32 patients. BMC Cancer. 2016;16:619.

17. Tahover E, Segal A, Isacson R, Rosengarten O, Grenader T, Gips M, Cherny N, Heching NI, Mesika L, Catane R, et al. Dexrazoxane added to doxorubicinbased adjuvant chemotherapy of breast cancer: a retrospective cohort study with a comparative analysis of toxicity and survival. Anti-Cancer Drugs. 2017;28(7):787-94.

18. Tian Z, Liu H, Zhang F, Li L, Du X, Li C, Yang J, Wang J. Retrospective review of the activity and safety of apatinib and anlotinib in patients with advanced osteosarcoma and soft tissue sarcoma. Investig New Drugs. 2020;38(5):155969.

19. Flannery $\mathrm{T}$, Kano H, Niranjan A, Monaco EA 3rd, Flickinger JC, Kofler J, Lunsford LD, Kondziolka D. Gamma knife radiosurgery as a therapeutic strategy for intracranial sarcomatous metastases. Int J Radiat Oncol Biol Phys. 2010;76(2):513-9.

20. Kim YA, Cho H, Lee N, Jung SY, Sim SH, Park IH, Lee S, Lee ES, Kim HJ. Doxorubicin-induced heart failure in cancer patients: a cohort study based on the Korean National Health Insurance Database. Cancer Med. 2018;7(12):6084-92.

21. Li J, Chang HM, Banchs J, Araujo DM, Hassan SA, Wagar EA, Yeh ETH, Meng $\mathrm{QH}$. Detection of subclinical cardiotoxicity in sarcoma patients receiving continuous doxorubicin infusion or pre-treatment with dexrazoxane before bolus doxorubicin. Cardiooncology. 2020;6:1.

22. Hequet O, Le QH, Moullet I, Pauli E, Salles G, Espinouse D, Dumontet C, Thieblemont C, Arnaud P, Antal D, et al. Subclinical late cardiomyopathy after doxorubicin therapy for lymphoma in adults. J Clin Oncol. 2004;22(10):1864-71.

23. Ewer MS, Von Hoff DD, Benjamin RS. A historical perspective of anthracycline cardiotoxicity. Heart Fail Clin. 2011;7(3):363-72.

24. Shee K, Kono AT, D'Anna SP, Seltzer MA, Lu X, Miller TW, Chamberlin MD. Maximizing the benefit-cost ratio of Anthracyclines in metastatic breast Cancer: case report of a patient with a complete response to high-dose doxorubicin. Case Rep Oncol. 2016;9(3):840-6.

25. Padegimas A, Clasen S, Ky B. Cardioprotective strategies to prevent breast cancer therapy-induced cardiotoxicity. Trends Cardiovasc Med. 2020:30(1):22-8.

26. Cardinale D, Colombo A, Bacchiani G, Tedeschi I, Meroni CA, Veglia F, Civelli M, Lamantia G, Colombo N, Curigliano G, et al. Early detection of anthracycline cardiotoxicity and improvement with heart failure therapy. Circulation. 2015;131(22):1981-8.

\section{Publisher's Note}

Springer Nature remains neutral with regard to jurisdictional claims in published maps and institutional affiliations. 